\title{
Análise comparativa da avaliação da lordose lombar aferida na radiografia em ortostase e na ressonância magnética
}

\author{
Cristina Saade Jaques', Lara Maria Miranda de Gouvêa', José Lucas Batista Júnior², \\ Charbel Jacob Junior', Igor Machado Cardoso², Adroaldo Nobrega Fonseca3, \\ Rafael Burgomeister Lourenço4, Rodrigo Rezende ${ }^{5}$
}

Escola Superior de Ciências da Santa Casa de Misericórdia de Vitória (Emescam), Vitória, ES, Brasil e Centro de Diagnóstico por Imagem (CDI), Vitória, ES, Brasil.

\section{RESUMO}

Objetivo: Comparar os ângulos encontrados na radiografia em ortostase e na ressonância magnética (RM) em decúbito dorsal com coxim sobre os membros inferiores. Métodos: Estudo prospectivo, de caráter descritivo, no qual se avaliou uma amostra de 100 pacientes, sendo 51 do sexo masculino, em que o grau de lordose lombar foi aferido por três examinadores independentes por meio da radiografia em ortostase e da RM em decúbito, com coxim, nos níveis de $L 1$ a $L 5$, de acordo com o método de Cobb. Resultados: A média dos valores encontrados na radiografia e na $R M$ com coxim de $L 1$ a $L 5$ foi de $42,2^{\circ}$ e $31,5^{\circ}$, respectivamente $(p<0,001)$, demonstrando que a angulação da lordose lombar aferida na RM subdimensiona a verdadeira angulação em $25 \%$. Conclusão: $A$ RM é insuficiente para a avaliação da lordose lombar, sendo de extrema importância a avaliação radiológica para avaliar a verdadeira angulação da lordose lombar.

\section{PALAVRAS-CHAVE}

Lordose, espectroscopia de ressonância magnética, radiografia.

\section{ABSTRACT}

Comparative analysis of assessment in computed radiography lumbar lordosis measured while standing and magnetic resonance imaging

Objective: To compare the angles found in radiography and magnetic resonance imaging (MRI) orthostatic supine with a pad on the lower limbs. Methods: Measurement of lumbar lordosis angles from 100 patients referred to MRI and computed radiography studies, using Cobb's method (with L1 and $L 5$ plateaus as reference). Measurements were done by three independent observers with different skills, and interobserver agreement was evaluated. The lumbar lordosis angle obtained in computed radiography and MRI were compared using statistical analysis with paired t-test. Results: The mean lordosis angles measured in computed radiography and MRI were respectively $42.2^{\circ}$ and $31.5^{\circ}$ $(p<0.001)$. Conclusion: There was good interobserver agreement. There was a statistically significant difference between the angles of lumbar lordosis in computed radiography and MRI. The data suggest that MRI is not reliable in the evaluation of the lumbar lordosis when used alone.

\section{KEYWORDS}

Lordosis, magnetic resonance spectroscopy, radiography.

\footnotetext{
Acadêmica do Curso de Medicina da Escola Superior de Ciências da Santa Casa de Misericórdia (Emescam), Vitória, ES, Brasil.

Médico-assistente do Grupo de Coluna Vertebral do Hospital Santa Casa de Misericórdia, Vitória, ES, Brasil.

Diretor Médico do Centro de Diagnóstico por Imagem (CDI), Vitória, ES, Brasil.

Radiologista do Centro de Diagnóstico por Imagem, Vitória, ES, Brasil.

5 Chefe do Grupo de Coluna Vertebral do Hospital Santa Casa de Misericórdia, Vitória, ES, Brasil.
} 


\section{Introdução}

A curvatura da coluna lombar apresenta relação com diversos fatores, como a curvatura torácica, a idade, o sexo e a inclinação pélvica, entre outros. ${ }^{1}$ Sua importância relaciona-se com o alinhamento vertebral, na medida em que esse é concebido como o resultado de um posicionamento lordótico ideal da vértebra, acima de uma pelve corretamente orientada. ${ }^{2}$

A lordose lombar consiste na curvatura fisiológica côncava posterior, localizada na região do segmento lombar da coluna vertebral, que termina no ângulo lombossacral formado na junção da vértebra L5 com o sacro. ${ }^{3}$ Ela é mantida basicamente por diferenças de espessura entre as partes anterior e posterior dos discos intervertebrais.

As alterações no alinhamento sagital podem conduzir a alterações da cinemática da coluna lombar. Isso pode posteriormente influenciar no suporte de carga e na distribuição de degeneração de disco intervertebral em cada nível da coluna, visto que o equilíbrio sagital lombar, as degenerações discais e a mobilidade segmentar têm influência recíproca um sobre o outro. ${ }^{4}$

A lordose lombar é avaliada ao exame físico como uma anteversão da pelve e desvio anterior da coluna no plano sagital, com ação dos músculos lombares e abdominais e eventuais desvios fisiológicos ou patológicos. No entanto, são necessários exames mais precisos da curvatura lombar para o diagnóstico correto do grau de lordose lombar.

O ângulo de lordose normal pode variar de $35^{\circ}$ até $50^{\circ}$, de acordo com a literatura ${ }^{5-8}$. O método de Cobb frequentemente é utilizado para medir o ângulo da curva lordótica por meio de radiografia simples de coluna com o paciente em ortostase, mas também é usualmente empregado na ressonância magnética (RM) da mesma forma para avaliação da lordose lombar.

Deve-se considerar, entretanto, que para o exame da curvatura lombar por meio da RM o paciente deve permanecer em decúbito dorsal e frequentemente com os membros inferiores fletidos sobre um coxim, visando a maior conforto para o paciente e redução da possibilidade de artefatos de mobilização. Essa posição implica extensão da coluna vertebral, com visível retificação da curvatura lombar. Portanto, a descrição pelo radiologista da angulação da lordose lombar aferida na RM pode criar um falso valor quando comparado às medidas realizadas na radiografia conforme descrito por Cobb.

Realizou-se o presente estudo no intuito de comparar os valores angulares da lordose lombar obtidos por meio de RM e das radiografias de coluna vertebral em ortostase.

\section{Casuística e métodos}

Estudo prospectivo, realizado após aprovação do Comitê de Ética em Pesquisa (CEP) da Santa Casa de Misericórdia de Vitória sob o número 12231813.6.0000.5065, no qual foram avaliados 100 pacientes. Os exames de imagem foram feitos no serviço do Centro de Diagnóstico por Imagem (CDI), por técnicos habilitados e laudados por radiologista especialista em doenças musculoesqueléticas do mesmo estabelecimento.

Utilizaram-se como critérios de inclusão pacientes portadores de lombalgia crônica que foram realizar exames de radiografia e ressonância magnética de coluna lombar sob prescrição médica.

Foram adotados como critérios de exclusão pacientes com menos de 20 anos de idade, grávidas, em período pós-operatório de cirurgia de coluna, com diagnóstico prévio de patologias vertebrais como espondilolistese, escoliose grave ou alterações congênitas, como vértebra de transição lombossacral. Também não foram avaliados pacientes cujos exames de imagem apresentaram platôs de L1 ou L5 não identificados.

A amostra obtida foi submetida à baixa radiação necessária para o exame de radiografia em posição ortostática e perfil. Em seguida, assumiu-se posição de decúbito dorsal com os membros inferiores em flexão sobre um coxim para a realização da RM.

O método padrão-ouro na literatura ${ }^{9}$ para obter a medida do ângulo da curvatura lordótica necessária para o diagnóstico preciso é o estudo radiográfico pelo método de Cobb, caracterizado por ter a mais alta confiabilidade intra- e interobservador. ${ }^{10} \mathrm{O}$ método supracitado consiste na medida da curva de lordose na incidência de perfil da coluna lombar, entre as vértebras L1 e L5. ${ }^{11}$

Os ângulos foram obtidos por imagens digitais por meio do uso de software de visualização clínica (OSIRIX, versão 5.5, Piximeo, Suíça), sendo realizados por três examinadores diferentes (dois com experiência inferior a um ano e um com experiência maior que um ano). As medidas foram realizadas de forma independente entre os examinadores, obedecendo a critérios previamente estabelecidos. Quando as medidas obtidas em determinado exame de imagem de um mesmo paciente variavam a partir de dez graus entre os examinadores, cada pesquisador realizou novo cálculo. Foi utilizada como parâmetro a superfície plana dos platôs vertebrais, excluindo-se eventuais espículas ou osteófitos anteriores e posteriores. ${ }^{12}$

Todos os dados avaliados apresentaram variáveis contínuas de distribuição normal, portanto, foram apresentados por meio da média, mediana, desvio-padrão, valor máximo e valor mínimo. 
Aplicou-se um teste de normalidade para avaliação das variáveis de cada paciente por pesquisador, sendo utilizado o teste de Shapiro-Wilk para verificar se os dados teriam uma distribuição normal Gaussiana.

Para avaliação da concordância entre os três pesquisadores, utilizou-se, para cada dois deles, o Coeficiente de Correlação Intraclasse (ICC) para a mensuração do grau de concordância ou confiabilidade das medidas realizadas entre dois observadores. Seu uso é indicado para amostra univariada e quando existem mais de dois grupos.

Como as medidas realizadas foram pareadas, por terem sido feitas nos mesmos indivíduos por métodos diferentes, justificou-se a utilização de testes pareados. Em decorrência da distribuição normal, aplicou-se o teste paramétrico de $t$ student pareado para se comparar as médias encontradas. Consideramos o valor de $p$ significativo quando este era menor que 0,050 entre as médias testadas.

\section{Resultados}

Dos 111 pacientes avaliados, 11 se enquadraram nos critérios de exclusão. Com isso, obteve-se uma amostra de 100 pacientes, sendo 51 do sexo masculino. A idade variou entre 20 e 76 anos em ambos os sexos, sendo a média de 46 anos.

Para avaliar a concordância entre os três pesquisadores, utilizou-se, para cada dois deles, o ICC, que demonstrou fidedignidade aceitável conforme tabela 1 .

De acordo com o maior tempo de experiência, foi escolhido o pesquisador 3 como sendo a referência padrão-ouro para a análise final dos resultados. Assim, a média dos valores angulares encontrados na radiografia e na RM com coxim de L1 a L5 foi de 42,2 e 31,5, respectivamente, conforme a tabela 2 .

Ao se analisar a correlação estatística entre as médias angulares da lordose lombar aferida na radiografia em ortostase e na RM lombar em decúbito, houve correlação estatisticamente diferente entre os valores conforme a tabela 3 .

Os resultados demonstraram que a medida da angulação da lordose lombar quando aferida na radiografia em ortostase é cerca de $25 \%$ maior que a medida aferida na RM lombar em decúbito dorsal com coxim.

\section{Discussão}

A lombalgia é uma condição com incidência e prevalência relativamente altas. Na maioria dos indivíduos acometidos, a dor lombar associada à incapacidade persiste por meses. Além disso, um pequeno percentual dos pacientes permanece com disfunção severa. Para aqueles em que a dor se resolve completamente, a recorrência em um ano não é incomum. ${ }^{13}$ Tudo isso contribui para a elevação do absenteísmo no trabalho e acarreta gastos com medicações, reabilitação e, em alguns casos, com tratamento cirúrgico. Sabe-se que a dor lombar crônica é uma condição persistente e de origem multifatorial ${ }^{14}$ e que o equilíbrio sagital adequado pode, em muito, contribuir para uma vida saudável sem dor lombar, portanto é imprescindível que o médico, durante a investigação diagnóstica de um paciente com lombalgia, solicite e avalie as curvaturas fisiológicas da coluna vertebral. Acontece que recentemente têm sido observadas aferições da angulação da lordose lombar realizada por meio da RM

\begin{tabular}{lccc}
\hline & \multicolumn{1}{c}{ Tabela 1 - Resultados do Coeficiente de Correlação Intraclasse (ICC) entre os pesquisadores } \\
\hline Variáveis & Pesquisador 1 x 2 & Pesquisador 1 x 3 & Pesquisador 2 x 3 \\
\hline RX L1-L5 & 0,981 & 0,975 & 0,975 \\
RM L1-L5 & 0,979 & 0,979 & 0,974 \\
\hline
\end{tabular}

\begin{tabular}{|c|c|c|c|c|c|c|c|}
\hline Pesquisador & Variáveis & $\mathbf{n}$ & Menor valor & Maior valor & Mediana & Média & Desvio-padrão \\
\hline \multirow[t]{2}{*}{ Pesquisador 3} & RX L1-L5 & 100 & 8,50 & 72,90 & 42,20 & 42,23 & 11,60 \\
\hline & RM L1-L5 & 100 & 5,40 & 64,60 & 32,40 & 31,56 & 11,34 \\
\hline
\end{tabular}

\begin{tabular}{lccc}
\hline & Tabela 3 - Resultados dos testes de comparação dos valores angulares & \\
\hline Número de pacientes & Média angular no RX L1-L5 & Média angular na RM L1-L5 & p-valor \\
\hline 100 & 42,23 & 31,56 & $<0,001$ \\
\hline
\end{tabular}


lombar em decúbito dorsal com utilização de coxins com objetivo de maior comodidade do paciente e de diminuir artefatos de posição.

Não foi encontrado na literatura nenhum trabalho que comparasse a angulação da lordose lombar aferida na radiografia em ortostase com a RM lombar. No presente estudo, observou-se maior valor angular nas radiografias do que na RM. Esta subdimensionou a angulação da lordose lombar em $25 \%$.

\section{Conclusão}

A radiografia lombar consiste no melhor método e o mais fidedigno exame a ser realizado na investigação diagnóstica das doenças degenerativas da coluna vertebral, pois, apesar de nos últimos tempos ter ocorrido grande avanço nos exames de imagem, não se pode diminuir a importância das radiografias e sim acrescentá-las no arsenal de exames complementares a ser realizado para um adequado diagnóstico e tratamento.

\section{Agradecimentos}

Sinceros agradecimentos em primeiro lugar a Deus e a todos aqueles que, de alguma forma, doaram um pouco de si para que a conclusão deste trabalho se tornasse possível.

A toda a equipe do Grupo de Coluna do Hospital Santa Casa de Misericórdia de Vitória e toda a equipe do Centro de Diagnóstico por Imagem de Vitória que nos acompanharam na concretização deste projeto.

Em especial aos doutores Rodrigo Rezende, in memoriam, e ao Rafael Lourenço, por todo auxílio, apoio e incentivo, sem os quais não teria sido possível a realização deste trabalho.

\section{Conflitos de interesse}

Os autores declaram não haver conflitos de interesse.

\section{Referências}

1. Damasceno LHF, Catarin SRG, Campos AD, Defino HLA. Lordose lombar: estudo dos valores angulares e da participação dos corpos vertebrais e discos intervertebrais. Acta Ortop Bras São Paulo. 2006;14(4):193-8.

2. Henneman AS, Antoneli PHL, Oliveira GC. Incidência pélvica: um parâmetro fundamental para definição do equilíbrio sagital da coluna vertebral. Coluna/Columna. 2012;11(3):237-9.

3. Moore KL, Dalley AF. Anatomia orientada para a clínica. $5^{\mathrm{a}}$ edição. Rio de Janeiro: Guanabara Koogan; 2007.

4. Keorochana G, Taghavi CE, Lee KB, Yoo JH, Liao JC, Fei $Z$, et al. Effect of sagittal alignment on kinematic changes and degree of disc degeneration in the lumbar spine: an analysis using positional MRI. Spine (Phila Pa 1976). 2011;36(11):893-8.

5. Abdel MP, Bodemer WS, Anderson PA. Supine thoracolumbar sagittal spine alignment: comparing computerized tomography and plain radiographs. Spine (Phila Pa 1976). 2012;37(4):340-5

6. Chen YL. Vertebral centroid measurement of lumbar lordosis compared with the Cobb technique. Spine (Phila Pa 1976). 1999;24(17):1786-90.

7. Fernand R, Fox DE. Evaluation of lumbar lordosis. A prospective and retrospective study. Spine (Phila Pa 1976). 1985;10(9):799-803.

8. Polly DW Jr, Kilkelly FX, McHale KA, Asplund LM, Mulligan $M$, Chang AS. Measurement of lumbar lordosis. Evaluation of intraobserver, interobserver, and technique variability. Spine (Phila Pa 1976). 1996;21(13):1530-5.

9. Harrison DE, Harrison DD, Cailliet R, Janik TJ, Holland B. Radiographic analysis of lumbar lordosis: centroid, Cobb, TRALL, and Harrison posterior tangent methods. Spine (Phila Pa 1976). 2001;26(11):E235-42.

10. Kuklo TR, Polly DW, Owens BD, Zeidman SM, Chang AS, Klemme WR. Measurement of thoracic and lumbar fracture kyphosis: evaluation of intraobserver, interobserver, and technique variability. Spine (Phila Pa 1976). 2001;26(1):61-5.

11. Hebert S, Xavier R, Pardini Junior AG, Filho TEPB. Ortopedia e traumatologia. $3^{a}$ edição. São Paulo: Artmed; 2003.

12. Keynan O, Fisher CG, Vaccaro A, Fehlings MG, Oner FC, Dietz J, et al. Radiographic measurement parameters in thoracolumbar fractures: a systematic review and consensus statement of the spine trauma study group. Spine (Phila Pa 1976). 2006;31(5):E156-65.

13. Koes BW, van Tulder M, Lin CW, Macedo LG, McAuley J, Maher C. An updated overview of clinical guidelines for the management of non-specific low back pain in primary care. Eur Spine J. 2010;19(12):2075-94.

14. Caby I, Olivier N, Mendelek F, Bou Kheir R, Vanvelcenaher J, Pelayo P. Functional restoration of the spine: effect of initial pain level on the performance of subjects with chronic low back pain. Pain Res Manag. 2014;19(5):E133-8.

Endereço para correspondência

Charbel Jacob Junior

Rua Desembargador Augusto Botelho, 33, ap. 203B

29101-110 - Vila Velha, ES, Brasil

E-mail: jcharbel@gmail.com 